Draft VERSion MARCh 31, 2020

Typeset using ${ }^{A} \mathrm{~T}_{\mathrm{E}} \mathrm{X}$ manuscript style in AASTeX63

\title{
Lithium in T Coronae Borealis
}

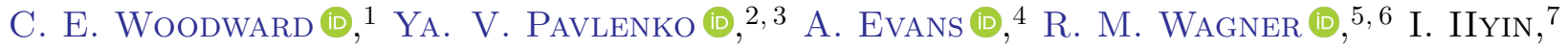
K. G. Strassmeier (D, ${ }^{7}$ S. Starrfield $\mathbb{D}^{8},{ }^{8}$ and U. Munari $\mathbb{D}^{9}$

${ }^{1}$ Minnesota Institute for Astrophysics, University of Minnesota, 116 Church Street SE, Minneapolis, MN 55455, USA

${ }^{2}$ Main Astronomical Observatory, Academy of Sciences of the Ukraine, Golosiiv Woods, Kyiv-127, 03680 Ukraine

${ }^{3}$ Centre for Astrophysics Research, University of Hertfordshire, College Lane, Hatfield, AL10 9AB, United Kingdom

${ }^{4}$ Astrophysics Group, Keele University, Keele, Staffordshire, ST5 5BG, UK

${ }^{5}$ Department of Astronomy, The Ohio State University, $140 \mathrm{~W}$. 18th Avenue, Columbus, OH 43210, USA

${ }^{6}$ Large Binocular Telescope Observatory, 933 North Cherry Avenue, Tucson, AZ 85721, USA

${ }^{7}$ Leibniz-Institut für Astrophysik Potsdam (AIP), An der Sternwarte 16, D-14482 Potsdam, Germany

${ }^{8}$ School of Earth and Space Exploration, Arizona State University, Box 871404, Tempe, AZ 85287-1404, USA

${ }^{9}$ INAF Astronomical Observatory of Padova, I-36012 Asiago (VI), Italy

(Received 2020 March 03; Revised March 31, 2020)

Submitted to AJ

\begin{abstract}
T Coronae Borealis ( $\mathrm{T} \mathrm{CrB}$ ) is a recurrent, symbiotic nova system currently in quiescence between its periodic $\approx 80$ yrs cycle of eruptions. Observations during interoutburst epochs provide an opportunity to study properties of the accretion disc and the $\mathrm{M}$ red giant. Here we present new irradiated (black body veiling) models, incorporating modern molecular opacities and line lists, of spectra derived from high resolution $(22,000 \lesssim \mathrm{R} \lesssim 120,000)$ optical echelle observations obtained at two epochs, one prior
\end{abstract}

Corresponding author: C.E. Woodward

chickw024@gmail.com 
to, and one post, the 2015 re-brightening event at similar spectroscopic system phase. We find a lithium abundance in the secondary at both epochs to be comparable. The non-irradiated (classical) model atmospheres yield a lithium abundance, $\mathrm{A}(\mathrm{Li})=1.3 \pm$ 0.1. The irradiated model (veiled) atmospheres, which are likely a better representation of the system in which the white dwarf and accretion disc illuminate the red giant, give $\mathrm{A}(\mathrm{Li})=2.4 \pm 0.1$

Keywords: Novae (1127): Recurrent Novae (1366): Astrochemistry (75): Chemical abundances (224): High resolution spectroscopy (2096)

\section{INTRODUCTION}

T Coronae Borealis ( $\mathrm{T} \mathrm{CrB})$ is a well-known member of the recurrent nova $(\mathrm{RN})$ class of objects. It is a binary system consisting of an MIII red giant (RG) and a white dwarf (WD) companion whose mass is close to the Chandrasekhar limit (see Kraft 1958; Shahbaz et al. 1997). Optical spectroscopy of the RG at quiescence reveals typical M-giant absorption features, with Balmer and He emission lines superimposed (see Kenyon \& Garcia 1986; Anupama \& Mikołajewska 1999; Mondal et al. 2020). It has undergone RN eruptions in 1866 and 1946.

In 2015, T CrB entered a high photometric state (Munari et al. 2016), with marked changes in the $V$ and $B$ light curves that persist to the present day. Comparison of the post-2015 photometric behavior with that immediately prior to the $1946 \mathrm{RN}$ eruption prompted Munari et al. to predict that the next RN eruption is imminent.

The RN eruptions in T CrB arise following a Thermonuclear Runaway (TNR) on the surface of the massive WD. Models of the RN eruption predict that substantial amounts of lithium may be produced (Hernanz et al. 1996; José \& Hernanz 1998; Starrfield et al. 1978, 2019). In T CrB as in all nova eruptions, material ejected by the WD as a result of a TNR is entrained by the atmosphere of the RG secondary.

The $\mathrm{Li}$ abundance, defined as $\mathrm{A}(\mathrm{Li})=12+\log [\mathrm{N}(\mathrm{Li}) / \mathrm{N}(\mathrm{H})]$ (Boesgaard et al. 2020, and references therein), of the $\mathrm{RG}$ in $\mathrm{T} \mathrm{CrB}$ has been considered by Shahbaz et al. 
(1999) and Wallerstein et al. (2008), who found (respectively) A(Li) = 0.6 and 0.8. However, these Li abundance analyses used computed spectra and conventional model atmospheres. The $\mathrm{T}$ CrB system contains, beside a RG, a WD and an accretion disk which can irradiate and affect the observed spectrum of the RG.

In this paper we combine the fits of the optical spectral energy distribution (SED) observed contemporaneously at low resolution $(R=\lambda / \Delta \lambda \simeq 2000)$ spanning from 4000 to $7000 \AA$, with that obtained at $R \simeq 120,000$ near the Li absorption lines at a comparable spectroscopic orbital phase. In particular we use irradiated (black body veiled) model atmospheres to derive Li abundances, and identify spectral characteristics of $\mathrm{T} \mathrm{CrB}$ in quiescence.

\section{OBSERVATIONS}

\subsection{The Multiple Mirror Telescope}

T CrB was observed at the Multiple Mirror Telescope (MMT; Beckers et al. 1981) 6.5-m on 2019 June 5.229 UT (JD = 2458639.7285) with the Blue Channel Spectrograph (Schmidt et al. 1989) with a thinned STA $2688 \times 512$ pixel detector spanning all or part of the $3800-7100 \AA$ region with a $3.6 \AA$ resolution. Observations were conducted with a $1^{\prime \prime} \times 180^{\prime \prime}$ long-slit aperture using a 500 line per mm grating in first order, with a tilt position of 2.033 , yielding a central wavelength position of $5505 \AA$. To prevent contamination in the red portion of the spectrum, second-order light was blocked using a UV-36 long-pass filter. HeArNe lamp spectra provided wavelength calibration and quartz-halogen lamp spectra provided flat-field correction images. Data were reduced using IRAF ${ }^{1}$ packages (Tody 1993, 1986), and standard spectra extraction and calibration techniques for optical data. Spectra of Kopff 27 (a spectrophotometric standard star) provided flux calibration. Eleven individual 5-s exposure spectra were extracted and co-added to produce the final spectra shown in Figure 1 . On June 5.229 UT, the system spectroscopic phase was 0.31 (conjunction of the M giant in front of the WD defined as phase $=0$ ) derived from the corrected photometric ephemerides of Lines et al. (1988)

${ }^{1}$ IRAF is distributed by the National Optical Astronomy Observatory, which is operated by the Association of Universities for Research in Astronomy (AURA) under a cooperative agreement with the National Science Foundation. 
as discussed in Belczynski \& Mikolajewska (1998). The observed spectrum used in our analysis was dereddened adopting an $\mathbf{E}(\mathbf{B}-\mathbf{V})=\mathbf{0 . 0 6}$.

\subsection{The Large Binocular Telescope}

We obtained high resolution optical spectroscopy of T CrB on 2019 June 21.1783 and 24.1782 UT (exposure mid-points of $\mathrm{JD}=2458655.6783$ and $\mathrm{JD}=2458658.6782$, respectively) with the $2 \times 8.4 \mathrm{~m}$ Large Binocular Telescope (LBT; Hill et al. 2008) using the Potsdam Echelle Polarimetric Spectroscopic Instrument (PEPSI; Strassmeier et al. 2015, 2018b). Our observations of T CrB used the $200 \mu \mathrm{m}$ diameter fiber, which projects a 1".5 diameter on the sky, and yields an instrumental spectral resolution $R \simeq 120,000$ with standard configuration for the image slicer. The cross-dispersers (CDs) combination (III $+\mathrm{V})$ was used on June 21 with a total integration time in each CD of 50 mins $(5$ exposures coadded, each $600 \mathrm{~s}$ in duration), while (III + VI) was used on June 24 with 78 mins of total integration time in each CD (3 coadded exposures). Spectra from both nights were combined using the PEPSI data reduction pipeline (see Strassmeier et al. 2018a) to produce a master spectrum covering a continuous wavelength range of 4800 to $5441 \AA$ and 6278 to $9067 \AA$, reaching a signal-to-noise (SNR) ratio per pixel greater than several hundred. A radial velocity correction of $-27.79 \mathrm{~km} \mathrm{~s}^{-1}$ was applied to correct to a heliocentric wavelength scale. The spectroscopic orbital phase for the PEPSI spectrum was $\sim 0.39$.

\subsection{Cima Ekar (Asiago)}

Prior to 2015, T CrB was in a low state of quiescent activity. High resolution spectra of $\mathrm{T}$ CrB were obtained on 1999 May 30.9113 UT (JD = 2451329.41126) with the REOSC Echelle spectrograph mounted on the Cima Ekar (Asiago) $1.82 \mathrm{~m}$ telescope. The REOSC is equipped with an Andor DW436-BV camera housing an E2V CCD42-40 AIMO CCD, $2048 \times 2048$ array, $13.5 \mu \mathrm{m}$ pixel, covering the interval $\lambda \lambda 3600$ to $7100 \AA$ in 30 orders, at a instrumental resolving power of 22000 for a 2 '.0 slit width, without inter-order wavelength gaps. The spectra were fully reduced in IRAF for bias, dark, flat, sky background, wavelength calibration and heliocentric correction. The continuum 
was normalized to 1.0 in each of the 30 individual orders prior to joining them into a single onedimensional (1D) spectrum. The spectroscopic orbital phase for the REOSC spectrum was $=0.20$.

\section{MODEL PROCEDURE}

One-dimensional (1D) SAM12 (Pavlenko 2003, 2006) was used to compute stellar atmospheres. A grid of theoretical synthetic RG spectra were computed for model atmospheres with effective temperatures $\left(T_{\text {eff }}\right)$ ranging from 3200 to $4000 \mathrm{~K}$ (see van Belle et al. 1999) with a step of $100 \mathrm{~K}$, a metallicity range of $0.0,-0.3,-0.6,-1.0$, and surface gravity, $\log g=1.0$. The latter value for the surface gravity is commensurate with that derived by Pavlenko et al. (2020). Direct comparison of the SAM12 and MARCS model atmospheres (see Gustafsson et al. 2008, and references therein) shows good agreement for their temperature structures, i.e., $\lesssim 50 \mathrm{~K}$, despite the differences in the adopted abundance scales.

Synthetic spectra, to compare with observations, were then computed from the stellar atmospheres using the program WITA6 (see Pavlenko et al. 1995, and references therein) assuming local thermodynamic equilibrium (LTE), hydro-static equilibrium and a one-dimensional (1D) model atmosphere without sources and sinks of energy. The model atmosphere and synthetic spectra assumed a stellar atmosphere microturbulent velocity $\left(V_{t}\right)$ of $3 \mathrm{~km} \mathrm{~s}^{-1}$ and the best fits to the observed T CrB spectra were determined by a $\chi^{2}$ minimization procedure as described by Pavlenko (2006, and references therein). Atomic lines were taken from VALD3 (Ryabchikova et al. 2015) and those for molecules $\mathrm{TiO}, \mathrm{VO}$ and $\mathrm{MgH}$ were obtained from different sources (for a more detailed discussion see Pavlenko 2014).

\section{RESULTS}

\subsection{The T CrB optical spectra at quiescence.}

Our identification of emission lines in the dereddened T CrB spectrum at quiescence, Figure 1, draws from tabulated lists from the NIST database (Kramida et al. 2019) and the $g_{k} * A_{i k}$ (where $g_{k}$ is the statistical weight and $A_{i k}$ is the transition probability) averaged air wavelengths compiled in 
the Atomic Line List website ${ }^{2}$ (for a description see van Hoof 2018), predicated by observations of known emission lines commonly seen in the spectra of various nova classes (Williams 2012). Strong hydrogen Balmer lines can be easily identified in the spectrum (Figure 1, top panel). The Balmer decrement is rather flat, suggesting H I comes from an extended high temperature shell. The He I lines are seen at $5876 \AA$ and $6678 \AA$. These lines form in hotter ionised gas $(\mathrm{T}>20,000 \mathrm{~K})$ than the H I. However, we do not see He I $\lambda 4471 \AA$ which usually can be observed in cataclysmic variable star (CVs) spectra (see Zwitter \& Munari 1995). Likely, He I forms under non-local thermodynamic equilibrium (NLTE), because the upper levels $E=186101.6 \mathrm{~cm}^{-1}$ and $191444.5 \mathrm{~cm}^{-1}$ of the $5876 \AA$ and $4471 \AA$ lines, respectively, do not differ much.

He II line emission is evident at $4686 \AA$. This line is of special interest due to the high ionization potential of He I $(24.6 \mathrm{eV})$ and the high excitation potential of the upper level of the corresponding He II transition $(48.4 \mathrm{eV})$. The line can be observed in spectra of cataclysmic binary stars (Sheets et al. 2007), in X-ray sources (Kaaret et al. 2004), including Cyg X-1 (Ninkov et al. 1987), in classical novae and older novae returning to quiescence (Williams 2012) and highly-ionized starburst regions in the extremely metal poor galaxies in the local Universe, like SBS 0335 - 052E (Kehrig et al. 2018). Clearly the presence in our spectrum of a strong He II line provides evidence for the existence of a very hot $(T \geq 60,000 \mathrm{~K})$ and extended shell or possibly a hot spot on, or near the WD surface.

Next we identify all spectral features (molecular bands) seen in absorption. The GAIA archive (Gaia Collaboration et al. 2016, 2018) cites an effective temperature of $3985 \mathrm{~K}$ for T CrB. However, synthetic spectra computed for $T_{\text {eff }}=4000 \mathrm{~K}$ do not satisfactorily reproduce the observed optical absorption features. Therefore we computed spectra for a cooler model atmosphere with $T_{\text {eff }}=$ $3800 \mathrm{~K}$ to aid in the identification of spectral features. We show results of comparison of our observed spectrum of $\mathrm{T} \mathrm{CrB}$ with theoretical spectra incorporating common hydrides, molecules, and other species seen in $M$ giant atmospheres (Figure 1 top panel). Titanium oxide ( $\mathrm{TiO})$ dominates the optical spectrum across a wide spectral range. These $\mathrm{TiO}$ features confirm that the RG is an M-

\footnotetext{
2 http://www.pa.uky.edu/ peter/newpage/
} 
giant and that its atmosphere has a $\mathrm{C} / \mathrm{O}$ ratio $\leq 1.0 . \mathrm{MgH}$ at $5200 \AA$ is still too strong in the $T_{\text {eff }}=$ $3800 \mathrm{~K}$ spectrum compared to the observed spectrum and we conclude that our $T_{\text {eff }}$ likely should be lower.

A fit of our synthetic spectra, computed with both $4000 \mathrm{~K},[\mathrm{Fe} / \mathrm{H}]=0.0$ (blue line) and a $3500 \mathrm{~K}$, $[\mathrm{Fe} / \mathrm{H}]=0.0$ (green line) classical 1D SAM12 model atmospheres, to the observed fluxes (red line) is shown in Figure 1, bottom panel. Clearly the $3500 \mathrm{~K},[\mathrm{Fe} / \mathrm{H}]=0.0$ model provides a better $\chi^{2}$-fit to the observed red part of the spectrum. Both models, however, are deficient at wavelengths bluewards of $\simeq 4300 \AA$. This suggests an additional emission component is required (§4.2).

\subsection{Irradiated spectra models}

To improve the fits, we invoke the presence of additional irradiation of the RG by the WD companion, and/or an accretion disc. Irradiation (veiling) effects were modeled by adding a contribution from a black body having a temperature $T_{\mathrm{bb}} \geq T_{\text {eff }}$ to the computed $\operatorname{spectra}\left(\mathbf{F}_{\text {irradiated }}\right)$ :

$$
F_{\text {irradiated }}=a * F_{\text {comp }}\left(T_{\text {eff }}\right)+(1-a) * F\left(T_{\mathrm{bb}}\right)
$$

where $F_{\text {comp }}$ is the flux computed for the classical model atmosphere, $a$ varies in the range of 0.6 to 1.0 , with a step size of 0.1 , and $F\left(T_{\mathrm{bb}}\right)$ is flux from a black body of temperature, $T_{\mathrm{bb}}(\mathrm{K})$. We computed a set of synthetic spectra for model atmospheres in the range $T_{\text {eff }}=3200$ to $4000 \mathrm{~K}$ in $100 \mathrm{~K}$ bins, $\log \mathrm{g}=1.0$, with black bodies of $T_{\mathrm{bb}}=5,000$ to $50,000 \mathrm{~K}$. To get the best fit to the observed spectrum we use our $\chi^{2}$ procedure (see Pavlenko 2006) defining a minimization function $S\left(=\sum_{n=1}^{i} s_{i}^{2}=\left|\left(F_{i}^{\mathrm{obs}}-F_{i}^{\mathrm{comp}}\right)\right|\right) . \quad S$ characterizes the averaged difference of fitted fluxes in one wavelength/frequency point and whose minimum value was found iteratively on the 3D grid of radial velocity sets, normalization factors, and broadening parameters. We also compute the errors of fits as the mean of the flux differences of computed and observed spectra, $\delta=\sum s_{i} / N$. To accelerate the iteration process, both theoretical and computed spectra are re-normalized to have a flux maximum of 1.0, as this simplifies the determination of the best fit. All fit spectra (observed vs. synthetic) shown within this manuscript therefore have flux ranges between 0 and 1.0. 
From this grid of models the best fit, yielding a value for the parameter $S=0.128 \pm 0.001$, is obtained for $T_{\text {eff }}=3500 \pm 100 \mathrm{~K}, T_{\mathrm{bb}}=8,000 \mathrm{~K},[\mathrm{Fe} / \mathrm{H}]=0.0$, and $a=0.8$ as shown in Figure 2.

To investigate the impact of RG gravity on our results, we repeat the computation with the same parameters, but with $\log g=0$. The best solution in this case is found for a synthetic spectrum of the form $0.8^{*} \mathrm{~F}(3500)+0.2^{*} T_{\mathrm{bb}}(8,000 \mathrm{~K})$ with $[\mathrm{Fe} / \mathrm{H}]=0.0$. Despite a larger value of $S=0.146 \pm 0.001 \mathrm{in}$ this case, we obtain a better solution for the spectral range around the $4200 \AA$ Ca I resonance line and at the red edge of the observed spectrum (Figure 3). The Ca I resonance line is pressure broadened and hence its profile shows a strong dependence on surface gravity. However, the spectrum at wavelengths near, and shortward of, the Ca I line is affected by the blue excess of the flux contributed by the WD and/or accretion disc complicating the analysis. Notwithstanding, we adopt a surface gravity (log g) equal to 1.0, as found by Pavlenko et al. (2020), in our subsequent analysis.

\subsection{Lithium in $\mathrm{T} C r B$}

The lithium abundance was determined by fitting our synthetic fluxes to the observed high resolution optical spectra. Synthetic spectra computations were carried out for a broad range of Li abundances $\mathrm{A}(\mathrm{Li})=0.2$ to 3.0. However, in modeling the high resolution spectra, we used a more extensive and detailed $\mathrm{TiO}$ line list taken from the molecular line list for exoplanets and other hot atmospheres (EXOMOL) database compilation (Tennyson et al. 2016; McKemmish et al. 2019) with solar isotopic ratios of $\mathrm{TiO}$. The best fit was determined by finding the minimum fit parameter $S$ over the spectral range from 6696 to $6725 \AA$. Our modeling considered only ${ }^{7} \mathrm{Li}$ lines (see Kurucz 1995; Mott et al. 2017), as the ${ }^{6} \mathrm{Li}$ abundances cannot be determined from our observed spectra which are broadened by strong microturbulence. Estimates of the ${ }^{7} \mathrm{Li} /{ }^{6} \mathbf{L i}$ ratio is challenging even in quieter, less convective solar-like stars (see Fig. 5 of Pavlenko et al. 2018). Interestingly, Mott et al. (2017) demonstrated that with high dispersion, high SNR ( 200$)$ spectra detection of ${ }^{6} \mathrm{Li}$ was possible in the spectrum of the giant star HD 123351, although absorption ascribed to ${ }^{6} \mathrm{Li}$ may have been mimicked by other effects, such as unknown weak blends, the Zeeman broadening, or asymmetric convection. 
Although a classical model atmosphere can be used, which was the technique applied in early works (such as Shahbaz et al. 1999; Wallerstein et al. 2008), a more robust fit to the observed spectrum in $\mathrm{T}$ CrB is achieved by using a model atmosphere with an irradiation term (Figure 2) as described in $§ 4.2$. We used both classical and irradiated models, however, to determine a lithium abundance from the fit to the 1999 REOSC spectrum (quiescence) and the more recent 2019 PEPSI spectrum obtained when T CrB was in a high state.

Generally, our results of lithium abundance determination depends on the accuracy of the TiO line lists. In the spectra of M-stars, lithium lines are seen on a background formed by a series of $\mathrm{TiO}$ absorption bands (see Figure 1 and Pavlenko et al. 1995, for more details). The continuum in T CrB cannot be defined in the observed spectrum, although we can use the pseudo-continuum formed by $\mathrm{TiO}$ bands as a proxy for the continuum when fitting the shape of the lithium absorption feature. Fortunately, modern $\mathrm{TiO}$ line lists allow us to provide reliable fits to the observed spectrum and the selected spectral features.

We carried out fits of our synthetic spectra to the observed spectrum across different spectral ranges 6700 to $6720 \AA$, and 6700 to $6715 \AA$. To remove from our analysis the effects of imperfect fitting of $\mathrm{TiO}$ features across spectral range of interest, we performed the lithium abundance determination in two steps. First, we determined the best fit of the $\mathrm{TiO}$ spectrum across the spectral range of the lithium doublet, i.e., 6700 to $6715 \AA$. Secondly, the part of lithium line profile which is not affected by $\mathrm{TiO}$ absorption was fit. Here we used the parameters of the minimization procedure determined in the prior step. The selected wavelength region of the lithium line profile used in this approach is located between two vertical pink lines in Figure 4 and Figure 5.

Our results are shown in the top panel of Figure 4, derived from the PEPSI spectra. We obtain $\mathrm{A}(\mathrm{Li})=1.2 \pm 0.1$ for the classical model atmosphere and $\mathrm{A}(\mathrm{Li})=2.4 \pm 0.1$ for the irradiated model atmosphere over both spectral ranges. The dependence of the minimization parameter $S$ on $\mathrm{A}(\mathrm{Li})$ is shown in the bottom panel of Figure 4. Our formal accuracy, \pm 0.1 dex, is determined by the extremely high sensitivity of the lithium line on $\mathrm{A}(\mathrm{Li})$ as shown in Figure 5. Modeling of the 1999 REOSC spectrum yields values for $\mathrm{A}(\mathrm{Li})$ of 1.4 and 2.2 for the classical and irradiated model 
atmospheres respectively. In the observed PEPSI spectrum of $\mathrm{T} \mathrm{CrB}$, the lithium line at $6708 \AA$ is not particularly strong.

Hydrodynamic studies (see Hernanz et al. 1996; José \& Hernanz 1998; Starrfield et al. 1978, 2019, and references therein) predicted that nova outbursts following TNRs on WDs are capable of significantly enriching the ejected gas with ${ }^{7}$ Be which decays to ${ }^{7} \mathrm{Li}$ (Bahcall \& Moeller 1969). These works confirmed recent optical spectroscopy of young nova systems at high dispersion (see Molaro et al. 2016; Wagner et al. 2018, and references therein). In RN systems, this Li also may contaminate the secondary. Alternatively, the $\mathrm{M}$ red giant in $\mathrm{T} \mathrm{CrB}$ may have enhanced Li abundance due to "dredge-up," as discussed by Charbonnel et al. (2020). The answer likely lies in ascertaining whether one detects ${ }^{7} \mathrm{Li}$ ("dredge-up" product) or ${ }^{6} \mathrm{Li}$ (spallation product) in the high dispersion spectra. The Cameron \& Fowler (1971) mechanism (in the late stages of stellar evolution) dredges up freshly synthesized ${ }^{7} \mathrm{Be}$ into the cooler surface regions of the star where it decays to ${ }^{7} \mathrm{Li}$ (Sackmann \& Boothroyd 1999). Mass loss from the progenitor of the T CrB WD could subsequently pollute the RG with ${ }^{7} \mathrm{Li}$.

In contrast, ${ }^{6} \mathrm{Li}$ could be produced by spallation reactions within the Asymptotic Giant Branch star (see Casuso \& Beckman 2000) or in the irradiated surface layers. However, evolution along the RG branch, where the convection zone mixing deepens, will preferentially destroy the more temperature sensitive ${ }^{6} \mathrm{Li}$ isotope relative to ${ }^{7} \mathrm{Li}$, and in any event leave little surface lithium. More intriguing is whether activity associated with the accretion disc, stellar flares (Ramaty et al. 2000), or TNR driven shocks could be the source of sufficient low-energy particles to drive creation of ${ }^{6} \mathrm{Li}$ (see Suzuki \& Inoue 2002). Nevertheless, given the microturbulent velocity of the RG in the T CrB system, any detection of ${ }^{6} \mathbf{L i}$, even with very high dispersion spectroscopy is unlikely.

The RG in $\mathrm{T} \mathrm{CrB}$ has undergone a long history of mass transfer between it and the more massive (and luminous) progenitor of the WD. Thus, the origin of the lithium excess is not clear. A determination of the ${ }^{7} \mathrm{Li}$ abundance immediately after the next outburst could clarify the source of ${ }^{7} \mathrm{Li}$. If the TNR produces gas enriched in ${ }^{7} \mathrm{Be}$ which 


\section{decays into ${ }^{7} \mathrm{Li}$ that is entrained by the $\mathrm{RG}$, a change in the surface ${ }^{7} \mathrm{Li}$ abundance might be evident.}

\subsection{Velocity Structure in Emission Lines}

The PEPSI spectra enable us to assess the velocity structure of strong $\mathrm{H} \alpha$ and $\mathrm{H} \beta$, and the weaker HeI and [O III] emission features with a precision of order $\lesssim 5 \mathrm{~km} \mathrm{~s}^{-1}$. Figure 6 shows two $200 \AA$ regions of interest near strong hydrogen recombination lines extracted from the continuum normalized PEPSI spectra of $\mathrm{T} \mathrm{CrB}$. Neither $\mathrm{H} \beta$ nor $\mathrm{H} \alpha$ exhibit the castellated line peaks often associated with velocity substructure arising from individual emission knots within the ejecta (Shore et al. 2016) and have profiles that can be well-fit by a single Gaussian. The full-width half-maximum velocities derived from fits to the line profiles are $\sim 142 \mathrm{~km} \mathrm{~s}^{-1}\left(\mathrm{EQW}_{\text {observed }}=-20.6 \AA\right)$ and $\sim 159 \mathrm{~km} \mathrm{~s}^{-1}\left(\mathrm{EQW}_{\text {observed }}\right.$ $=-34.5 \AA$ ) for $\mathrm{H} \beta$ and $\mathrm{H} \alpha$ respectively. Balmer line profiles in symbiotic stars are dominated by the geometry of the ionized fraction of the RG wind. Superimposed absorption components arise from external and neutral zones of the winds, with broad wings from high electronic pressure, orbital visibility of the colliding winds zone near the inner Lagrangian points and hot spot(s) (Shore et al. 2012; Munari \& Banerjee 2018). No evidence for He II $\left(\lambda_{\text {air }}=6560.097 \AA\right)$ is evident on the blue wing of $\mathrm{H} \alpha$. Both [O III] and He I have doubly peaked emission profiles, with velocity components (with respect to the rest wavelengths) of -20.8 and $+15.0 \mathrm{~km} \mathrm{~s}^{-1}$ for [O III] and -62.9 and $+52.3 \mathrm{~km} \mathrm{~s}^{-1}$ for He I. The velocity components come from the accretion disc, but further phase resolved studies are required to confirm this conjecture.

\section{CONCLUSION}

High dispersion optical spectroscopy of $\mathrm{T} \mathrm{CrB}$, a recurrent symbiotic-like nova (recurrence period $\approx 80$ yrs) currently in quiescence, was analyzed with a grid of state-of-the-art model atmospheres that incorporate modern molecular line lists, (including high accuracy TiO line lists), computed over a range of $[\mathrm{Fe} / \mathrm{H}]$ yielding new determinations of the lithium abundance of red giant (MIII) atmospheres. 
A classical 1-dimensional model atmosphere analysis of the $\mathrm{T}$ CrB system yields $\mathrm{A}(\mathrm{Li})=1.2 \pm 0.1$, while a model atmosphere that includes irradiation (black body veiling) by the white dwarf and/or accretion disc results in $\mathrm{A}(\mathrm{Li})=2.4 \pm 0.1$. In both cases, the best fits were found with $[\mathrm{Fe} / \mathrm{H}]=0.0$ and with a surface gravity $(\log g)$ equal to 1.0. The latter atmosphere is likely more representative of the irradiation in the system which is complex. The clearly visible blue excess shortward of $4300 \AA$ is likely a signature of the hot white dwarf and accretion disc. The presence of He II suggests a hot outer envelope. The necessity of adding an additional irradiation term (black body veiling) to correctly model the continuum suggests that extra heating components in the system are required.

Our lithium abundances will serve as a baseline for comparison to those determined during the next nova outburst of the system to ascertain whether enhancement due to pollution can occur. In this scenario, the nova outburst destroys ${ }^{7} \mathrm{Li}$ accreted from the secondary, but produces new ${ }^{7} \mathrm{Li}$ in the thermonuclear runaway. The white dwarf ejecta will entrain both the material from the secondary and also surrounding gas enhanced with ${ }^{7} \mathrm{Li}$ from the red giant. 


\section{ACKNOWLEDGMENTS}

The authors thank the referee for their insightful comments and suggestions that improved the manuscript. The LBT is an international collaboration among institutions in the United States, Italy and Germany. LBT Corporation partners are: The University of Arizona on behalf of the Arizona Board of Regents; Istituto Nazionale di Astrofisica, Italy; LBT Beteiligungsgesellschaft, Germany, representing the Max-Planck Society, The Leibniz Institute for Astrophysics Potsdam, and Heidelberg University; The Ohio State University, and collaborating institutions The University of Notre Dame, University of Minnesota and University of Virginia. Observations reported here were also obtained at the MMT Observatory, a joint facility of the Smithsonian Institution and the University of Arizona. This work also has made use of data from the European Space Agency (ESA) mission Gaia (https://www.cosmos.esa.int/gaia), processed by the Gaia Data Processing and Analysis Consortium (DPAC, https://www.cosmos.esa.int/web/gaia/dpac/consortium). Funding for the DPAC has been provided by national institutions, in particular the institutions participating in the Gaia Multilateral Agreement. CEW acknowledges partial support from NASA 80NSSC19K0868. YP's work was funded as part of the routine financing program for institutes of the National Academy of Sciences of Ukraine. SS gratefully acknowledges partial support from NSF Grant AST1107484 and NASA Theory Grant 14-ATP14-0007 to ASU along with support from ASU through his Regents' Professor monies.

Facilities: LBT (PEPSI) (Hill et al. 2008; Strassmeier et al. 2015), MMT (BlueChannel) (Beckers et al. 1981; Schmidt et al. 1989), Asiago (Echelle), GAIA (Gaia Collaboration et al. 2016)

Software: IRAF (Tody 1993, 1986), SAM12 (Pavlenko 2003, 2006), WITA6 (Pavlenko et al. 1995) 


\section{REFERENCES}

Anupama, G. C., \& Mikołajewska, J. 1999, A\&A, 324 $344,177$.

https://arxiv.org/abs/astro-ph/9812432

325

326

Bahcall, J. N., \& Moeller, C. P. 1969, ApJ, 155, 327 511, doi: 10.1086/149887

Beckers, J. M., Ulich, B. L., Shannon, R. R., et al ${ }_{329}$ 1981, The Multiple Mirror Telescope., ed. 330

G. Burbidge \& A. Hewitt, $63-128$

Belczynski, K., \& Mikolajewska, J. 1998, MNRAS $_{332}$ 296, 77, doi: 10.1046/j.1365-8711.1998.01301.x

Boesgaard, A. M., Lum, M. G., \& Deliyannis,

C. P. 2020 , ApJ, 888,28 ,

doi: 10.3847/1538-4357/ab4fdb

Cameron, A. G. W., \& Fowler, W. A. 1971, ApJ, 164, 111, doi: 10.1086/150821

Casuso, E., \& Beckman, J. E. 2000, PASP, 112, 942, doi: 10.1086/316592

Charbonnel, C., Lagarde, N., Jasniewicz, G., et al. 2020, A\&A, 633, A34,

doi: 10.1051/0004-6361/201936360

Gaia Collaboration, Prusti, T., de Bruijne,

J. H. J., et al. 2016, A\&A, 595, A1, doi: 10.1051/0004-6361/201629272

Gaia Collaboration, Brown, A. G. A., Vallenari, A., et al. 2018, A\&A, 616, A1, doi: 10.1051/0004-6361/201833051

Gustafsson, B., Edvardsson, B., Eriksson, K., $\quad 349$ et al. 2008, A\&A, 486, 951, doi: 10.1051/0004-6361:200809724

Hernanz, M., Jose, J., Coc, A., \& Isern, J. 1996, 352 ApJL, 465, L27, doi: 10.1086/310122
Hill, J. M., Green, R. F., Slagle, J. H., et al. 2008, Society of Photo-Optical Instrumentation Engineers (SPIE) Conference Series, Vol. 7012, The Large Binocular Telescope, 701203, doi: $10.1117 / 12.790065$

José, J., \& Hernanz, M. 1998, ApJ, 494, 680, doi: $10.1086 / 305244$

Kaaret, P., Ward, M. J., \& Zezas, A. 2004, MNRAS, 351, L83, doi: 10.1111/j.1365-2966.2004.08020.x

Kehrig, C., Vílchez, J. M., Guerrero, M. A., et al. 2018, MNRAS, 480, 1081, doi: 10.1093/mnras/sty1920

Kenyon, S. J., \& Garcia, M. R. 1986, AJ, 91, 125, doi: 10.1086/113991

Kraft, R. P. 1958, ApJ, 127, 625, doi: 10.1086/146495

Kramida, A., Yu. Ralchenko, Reader, J., \& and NIST ASD Team. 2019, NIST Atomic Spectra Database (ver. 5.7.1), [Online]. Available: https://physics.nist.gov/asd [2019, November 22]. National Institute of Standards and Technology, Gaithersburg, MD.

Kurucz, R. L. 1995, ApJ, 452, 102, doi: $10.1086 / 176283$

Lines, H. C., Lines, R. D., \& McFaul, T. G. 1988, AJ, 95, 1505, doi: 10.1086/114746

McKemmish, L. K., Masseron, T., Hoeijmakers, H. J., et al. 2019, MNRAS, 488, 2836, doi: $10.1093 / \mathrm{mnras} / \mathrm{stz} 1818$ 
Molaro, P., Izzo, L., Mason, E., Bonifacio, P., \& 385 Della Valle, M. 2016, MNRAS, 463, L117, 386 doi: $10.1093 / \mathrm{mnrasl} / \mathrm{slw} 169$ 387

Mondal, A., Das, R., Anupama, G. C., \& Mondal $_{388}$ S. 2020, MNRAS, 492, 2326, doi: 10.1093/mnras/stz3570

Mott, A., Steffen, M., Caffau, E., Spada, F., \& 391 Strassmeier, K. G. 2017, A\&A, 604, A44, doi: 10.1051/0004-6361/201730409

Munari, U., \& Banerjee, D. P. K. 2018, MNRAS, 394 475, 508, doi: 10.1093/mnras/stx3192

Munari, U., Dallaporta, S., \& Cherini, G. 2016, 396 NewA, 47, 7, doi: 10.1016/j.newast.2016.01.002 397

Ninkov, Z., Walker, G. A. H., \& Yang, S. 1987, 398 ApJ, 321, 438, doi: 10.1086/165642

Pavlenko, Y. V. 2003, Astronomy Reports, 47, 59,400 doi: $10.1134 / 1.1538496$

—. 2006, Mem. Soc. Astron. Italiana, 77, 1002. 402 https://arxiv.org/abs/astro-ph/0606252 403

—. 2014, Astronomy Reports, 58, 825, doi: 10.1134/S1063772914110043

Pavlenko, Y. V., Banerjee, D. P. K., Evans, A., ${ }^{406}$ et al. 2020, MNRAS, submitted

Pavlenko, Y. V., Jenkins, J. S., Ivanyuk, O. M., 408 et al. 2018, A\&A, 611, A27, doi: 10.1051/0004-6361/201731547

Pavlenko, Y. V., Rebolo, R., Martin, E. L., \& Garcia Lopez, R. J. 1995, A\&A, 303, 807

Ramaty, R., Tatischeff, V., Thibaud, J. P., Kozlovsky, B., \& Mandzhavidze, N. 2000, ApJL, 534, L207, doi: 10.1086/312671
Ryabchikova, T., Piskunov, N., Kurucz, R. L., et al. 2015, PhyS, 90, 054005, doi: 10.1088/0031-8949/90/5/054005

Sackmann, I. J., \& Boothroyd, A. I. 1999, ApJ, 510, 217, doi: 10.1086/306545

Schmidt, G. D., Weymann, R. J., \& Foltz, C. B. 1989, PASP, 101, 713, doi: 10.1086/132495

Shahbaz, T., Hauschildt, P. H., Naylor, T., \& Ringwald, F. 1999, MNRAS, 306, 675, doi: 10.1046/j.1365-8711.1999.02549.x

Shahbaz, T., Somers, M., Yudin, B., \& Naylor, T. 1997, MNRAS, 288, 1027, doi: 10.1093/mnras/288.4.1027

Sheets, H. A., Thorstensen, J. R., Peters, C. J., Kapusta, A. B., \& Taylor, C. J. 2007, PASP, 119, 494, doi: 10.1086/518698

Shore, S. N., Wahlgren, G. M., Augusteijn, T., et al. 2012, A\&A, 540, A55, doi: 10.1051/0004-6361/201118060

Shore, S. N., Mason, E., Schwarz, G. J., et al. 2016, A\&A, 590, A123, doi: 10.1051/0004-6361/201527856

Starrfield, S., Bose, M., Iliadis, C., et al. 2019, arXiv e-prints, arXiv:1910.00575. https://arxiv.org/abs/1910.00575

Starrfield, S., Truran, J. W., Sparks, W. M., \& Arnould, M. 1978, ApJ, 222, 600, doi: $10.1086 / 156175$

Strassmeier, K. G., Ilyin, I., \& Steffen, M. 2018a, A\&A, 612, A44, doi: 10.1051/0004-6361/201731631 
Strassmeier, K. G., Ilyin, I., Järvinen, A., et al. ${ }_{438}$ 2015, Astronomische Nachrichten, 336, 324, 439 doi: 10.1002/asna.201512172

Strassmeier, K. G., Ilyin, I., Weber, M., et al. 2018b, in Society of Photo-Optical Instrumentation Engineers (SPIE) Conference ${ }_{442}$ Series, Vol. 10702, Proc. SPIE, 1070212, doi: $10.1117 / 12.2311627$

Suzuki, T. K., \& Inoue, S. 2002, ApJ, 573, 168, ${ }_{445}$ doi: $10.1086 / 340487$

Tennyson, J., Yurchenko, S. N., Al-Refaie, A. F., et al. 2016, Journal of Molecular Spectroscopy, 447 327, 73, doi: 10.1016/j.jms.2016.05.002 448

Tody, D. 1986, Society of Photo-Optical Instrumentation Engineers (SPIE) Conference Series, Vol. 627, The IRAF Data Reduction and ${ }^{450}$ Analysis System, ed. D. L. Crawford, 733, doi: $10.1117 / 12.968154$

—. 1993, Astronomical Society of the Pacific

Conference Series, Vol. 52, IRAF in the Nineties, ed. R. J. Hanisch, R. J. V. Brissenden, \& J. Barnes, 173 van Belle, G. T., Lane, B. F., Thompson, R. R., et al. 1999, AJ, 117, 521, doi: 10.1086/300677

van Hoof, P. A. M. 2018, Galaxies, 6, 63, doi: 10.3390/galaxies6020063

Wagner, R. M., Woodward, C. E., Starrfield, S., Ilyin, I., \& Strassmeier, K. 2018, in American Astronomical Society Meeting Abstracts, Vol. 231, American Astronomical Society Meeting Abstracts \#231, 358.10

Wallerstein, G., Harrison, T., Munari, U., \& Vanture, A. 2008, PASP, 120, 492, doi: $10.1086 / 587965$

Williams, R. 2012, AJ, 144, 98, doi: 10.1088/0004-6256/144/4/98

Zwitter, T., \& Munari, U. 1995, A\&AS, 114, 575 


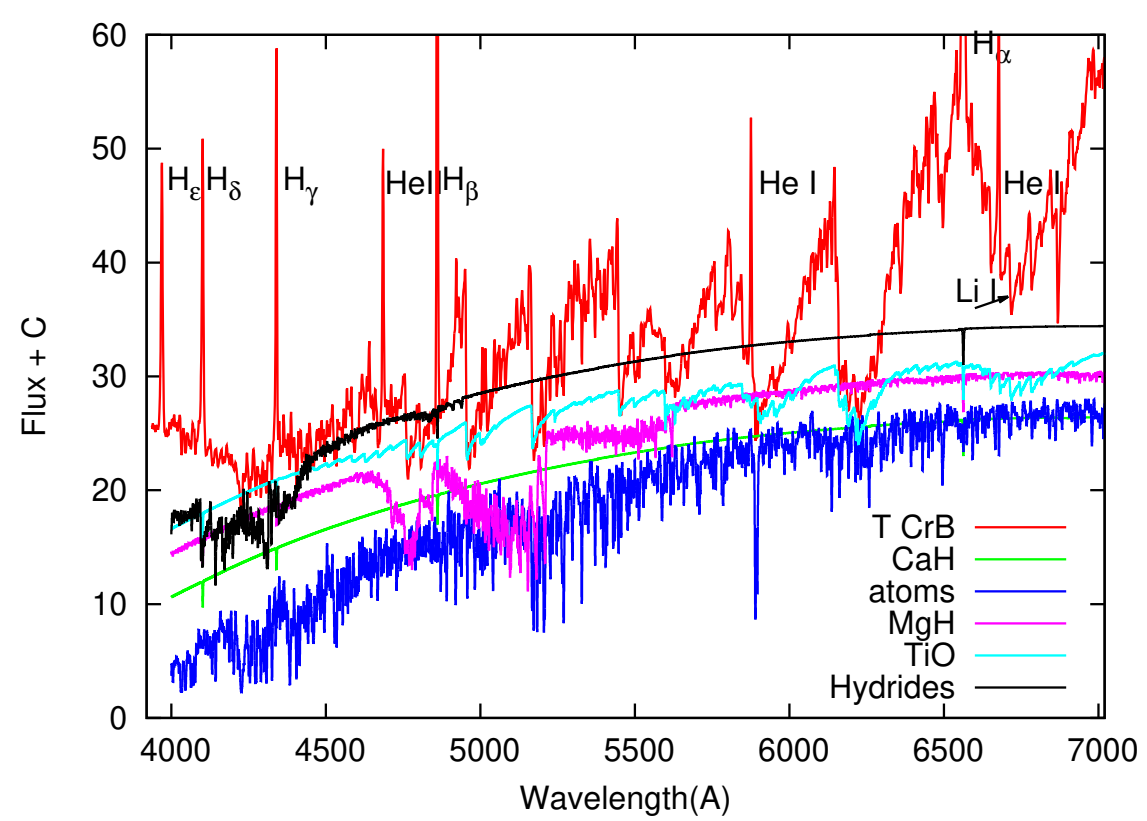

(a)

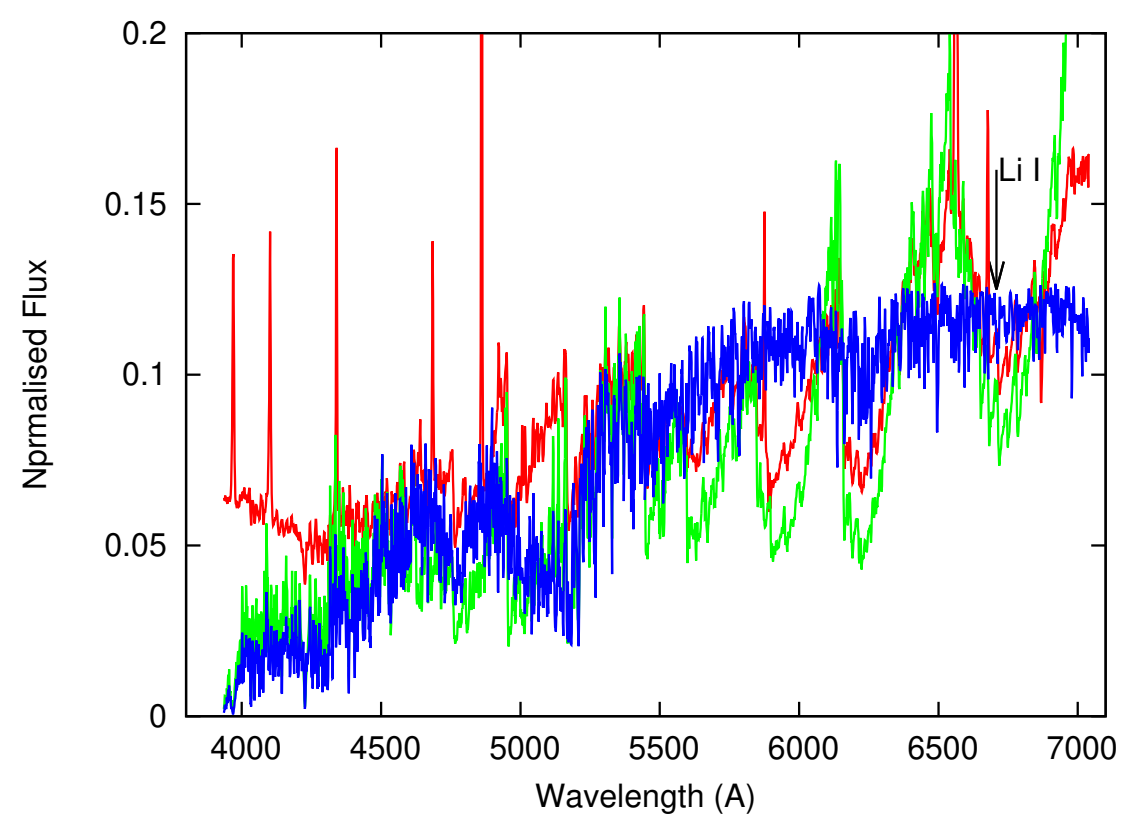

(b)

Figure 1. The MMT optical spectrum of T CrB obtained on 2019 June 05.485 UT, dereddened with an $\mathrm{E}(\mathrm{B}-\mathrm{V})=0.06$. (a) Top: Identifications of the main emission and absorption features in the observed spectrum (red line). Contributions of various hydrides, molecules, and other species to the observed spectral energy distribution are also shown (individual color coded curves). Clearly seen at $\lambda \leq 4200 \AA$ is a red tail of UV excess, produced by a hot spot, white dwarf, or accretion disc (or a combination of the latter). (b) Bottom: The observed spectrum (red line) fit with synthetic spectra computed with a classical 1D model atmosphere with a $T_{\text {eff }}=3500$ (green line) and $T_{\text {eff }}=4000$ (blue line). In both $T_{\text {eff }}$ cases, $\log \mathrm{g}=1.0$ and $[\mathrm{Fe} / \mathrm{H}]=0.0$. 


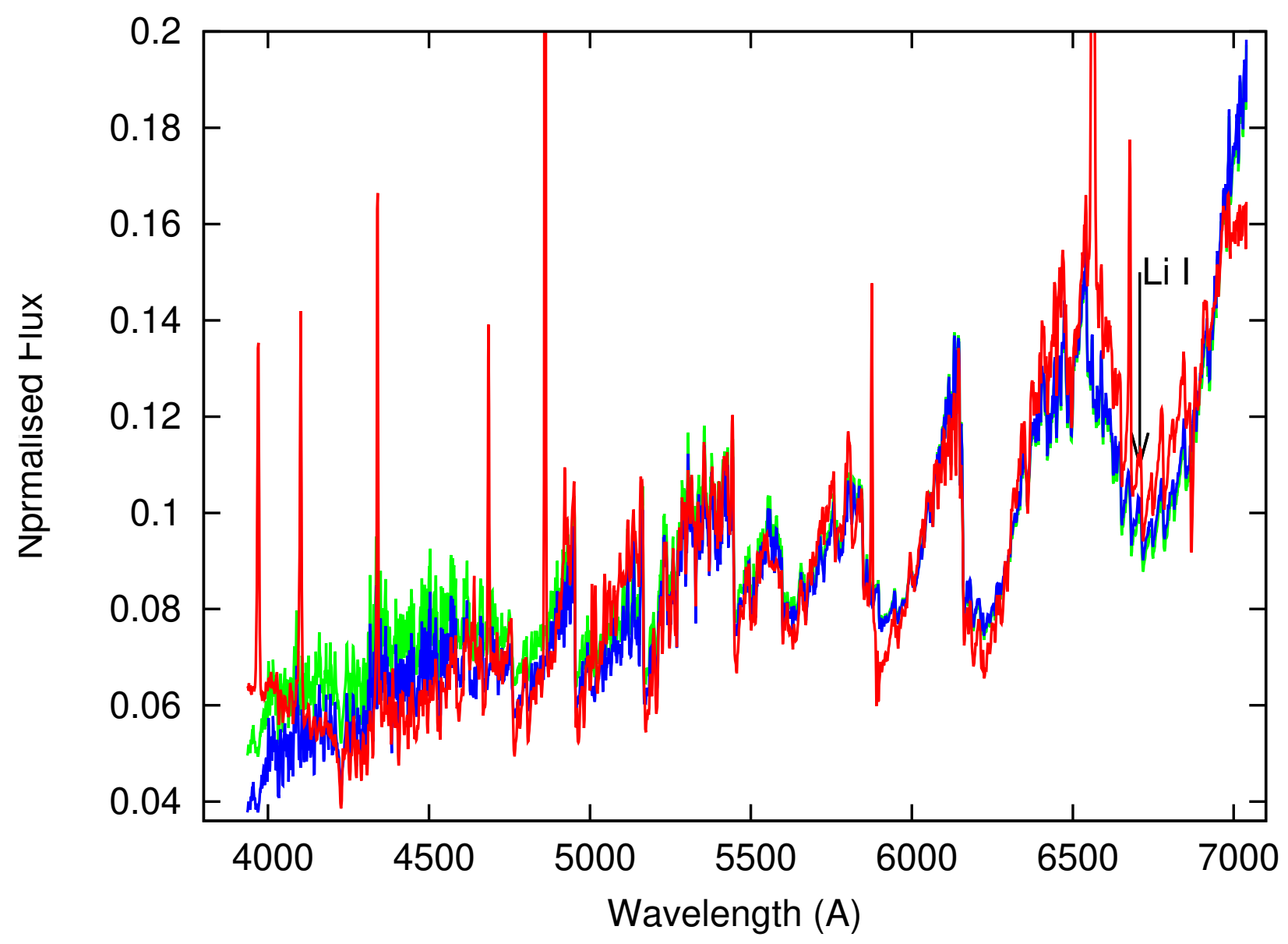

Figure 2. The observed, dereddend T CrB 2019 June MMT optical spectrum (red line) fit with a synthetic irradiated (black body veiled) spectra of the form $F_{\text {irradiated }}=a * F_{\text {comp }}\left(T_{\text {eff }}\right)+(1-a) * F\left(T_{\mathrm{bb}}\right)$. A model with $T_{\text {eff }}=3500 \mathrm{~K}, T_{\mathrm{bb}}=10,000 \mathrm{~K}$ is given by the green line, while the blue line is a model with $T_{\text {eff }}=3500 \mathrm{~K}$ and $T_{\mathrm{bb}}=8.000 \mathrm{~K}$. For both cases $\log \mathrm{g}=1.0,[\mathrm{Fe} / \mathrm{H}]=0.0$, and $a=0.8$. The position of the lithium absorption feature is indicated. 


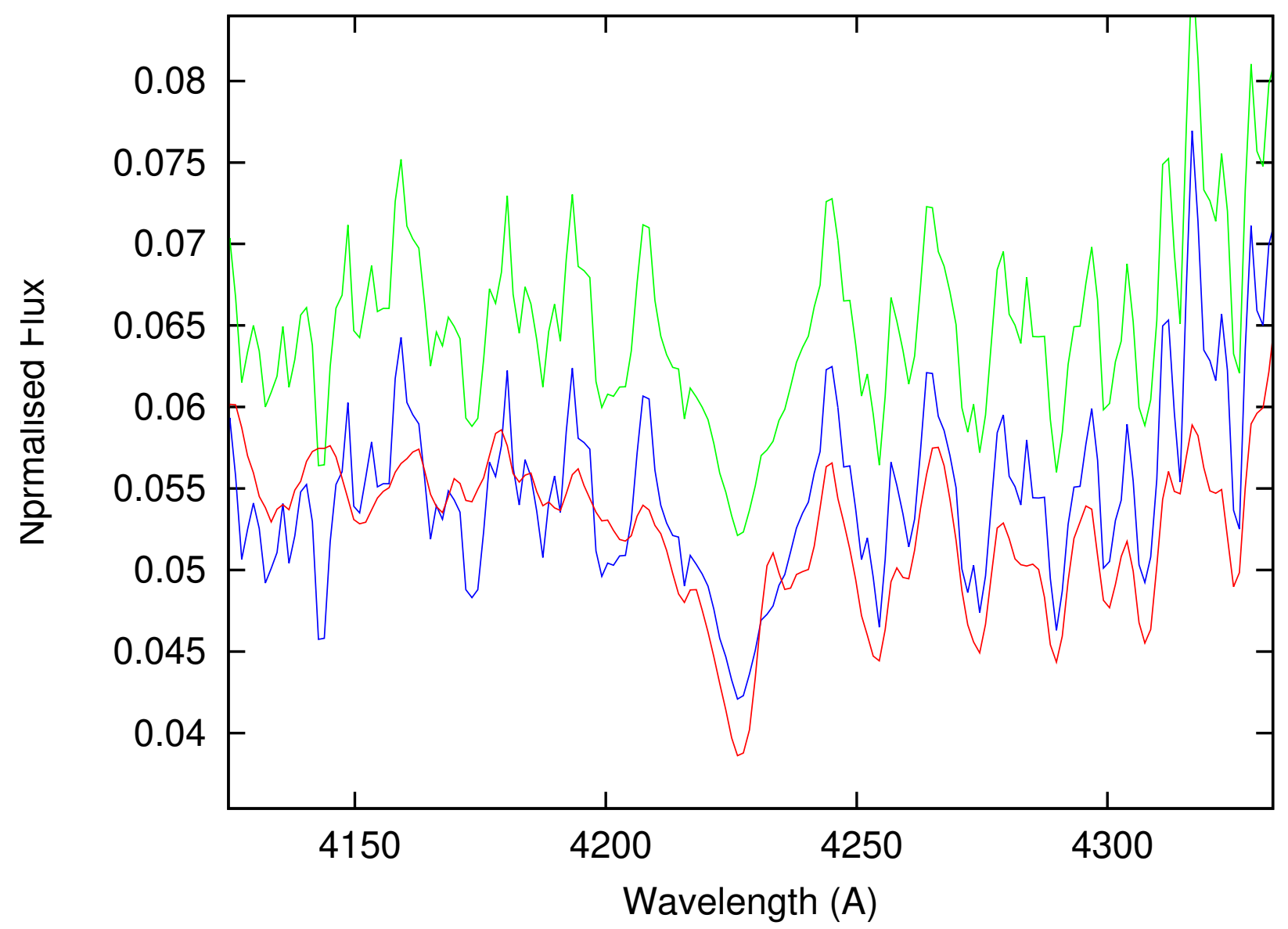

Figure 3. The $4226.7 \AA$ Ca I resonance line in T CrB observed in the 2019 June MMT optical spectrum (red line) fit with a synthetic irradiated (black body veiled) spectra as described in Figure 2. The model color coding is the same as Figure 2. 


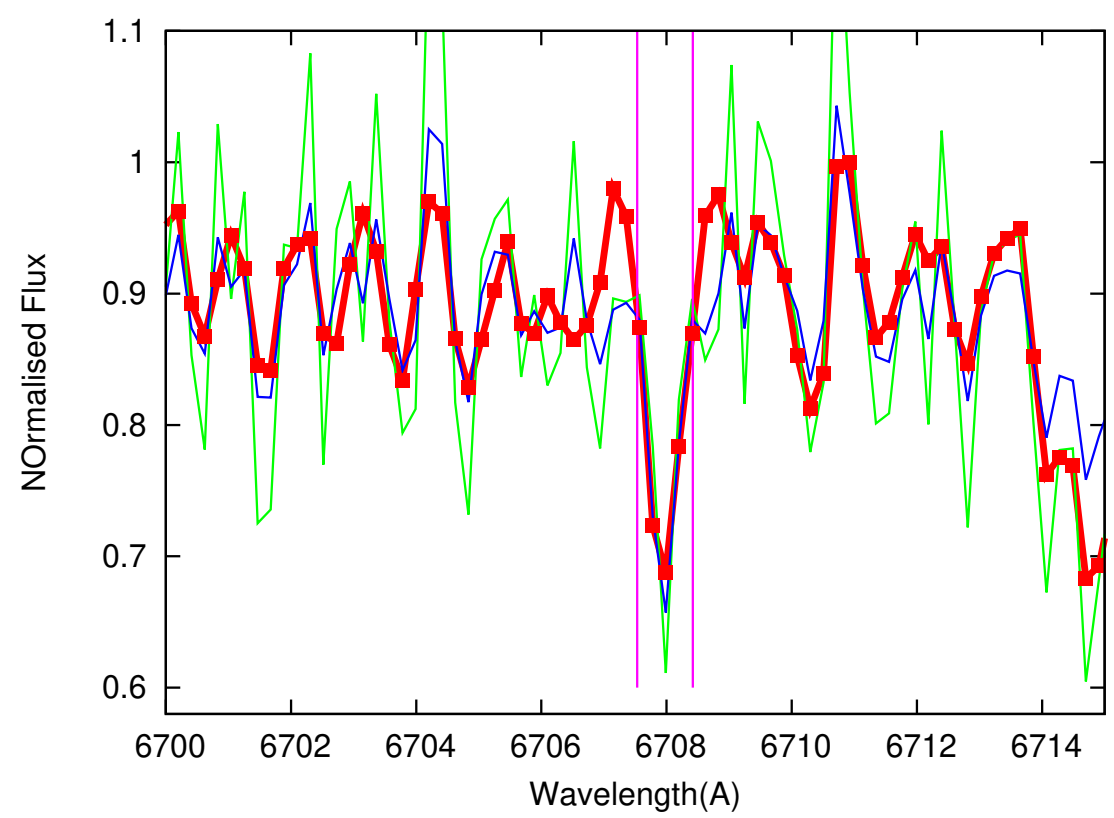

(a)

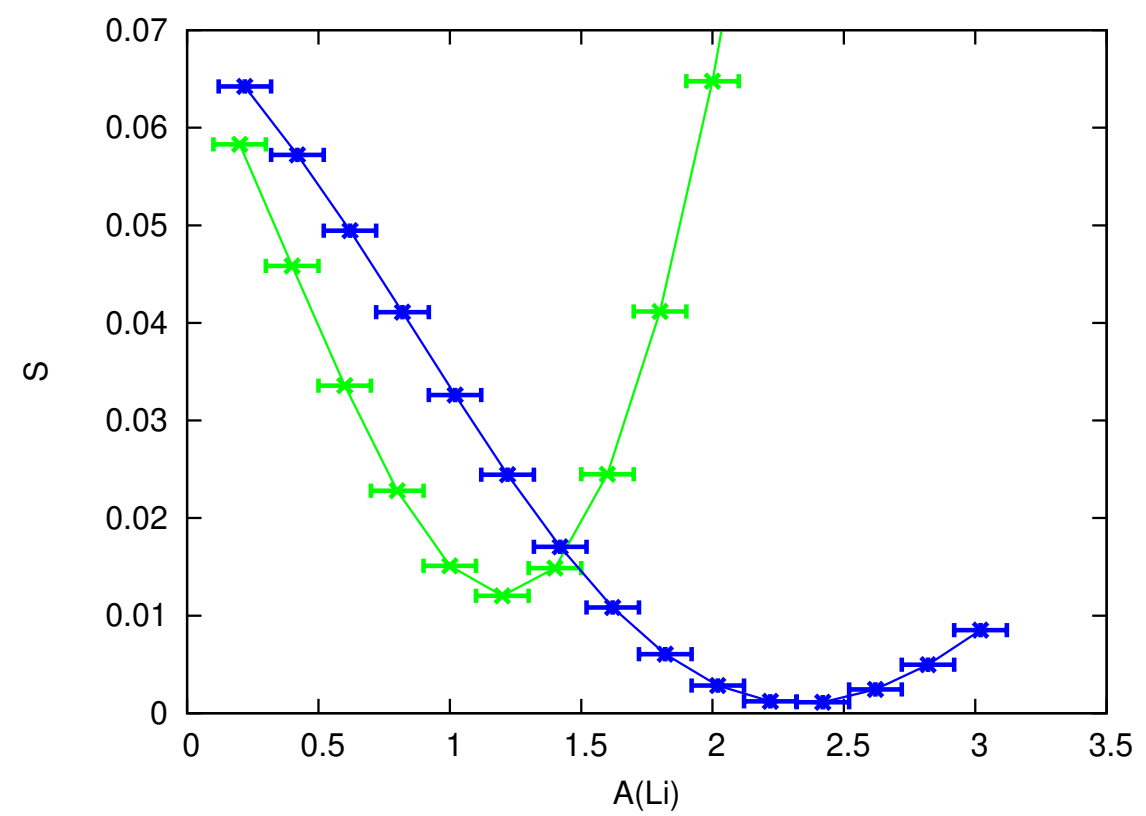

(b)

Figure 4. (a) Top: Fits to the the observed $6708 \AA \mathrm{Li}$ I resonance doublet in $\mathrm{T} \mathrm{CrB}$ (red line) which is highly broadened by macro-turbulence. We use a classical 1D model atmosphere (green line) and and irradiated (blue line) model (described in Figure 2) which yields a best-fit values of the lithium abundance, $\mathrm{A}(\mathrm{Li})=1.2$ and 2.4 , respectively. The spectral region used in the model fitted which contains the $\mathrm{Li}$ I doublet is marked by the vertical pink lines. (b) Bottom: The dependence of the model minimization parameter $S$ on $\mathrm{A}(\mathrm{Li})$ for the classical (green line) and the irradiated model (blue line) cases. 


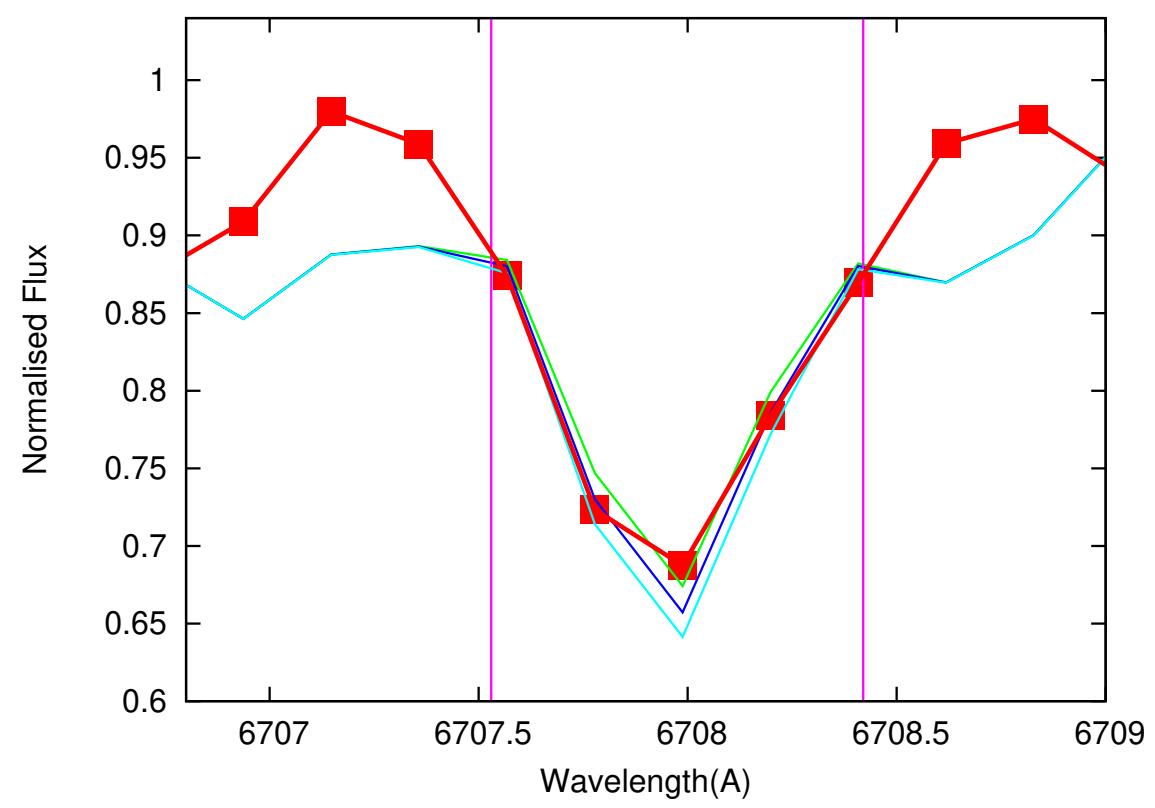

Figure 5. An enlarged view of the observed $6708 \AA \mathrm{Li}$ I doublet line profile (red line) in $\mathrm{T}$ CrB from the 2019 June PEPSI data (Figure 4) compared with computed irradiated (black body veiled) model for three lithium abundances, $\mathrm{A}(\mathrm{Li})=2.2$ (green line), 2.4 (blue line), and 2.6 (cyan line). 


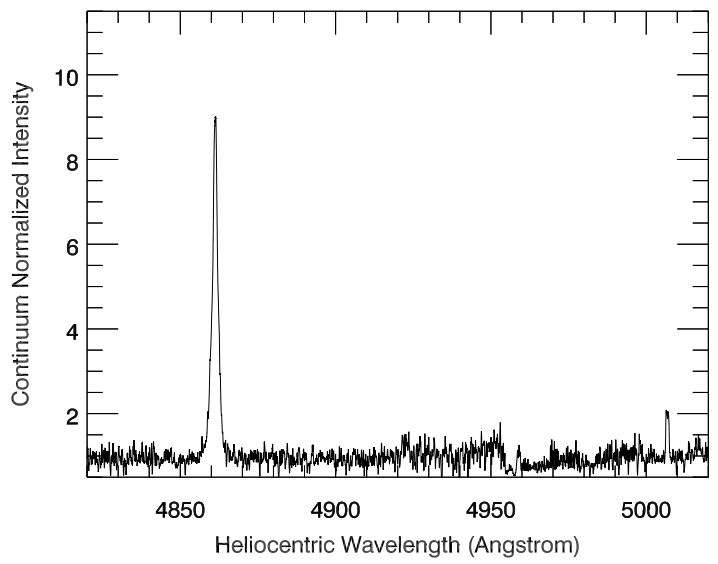

(a)

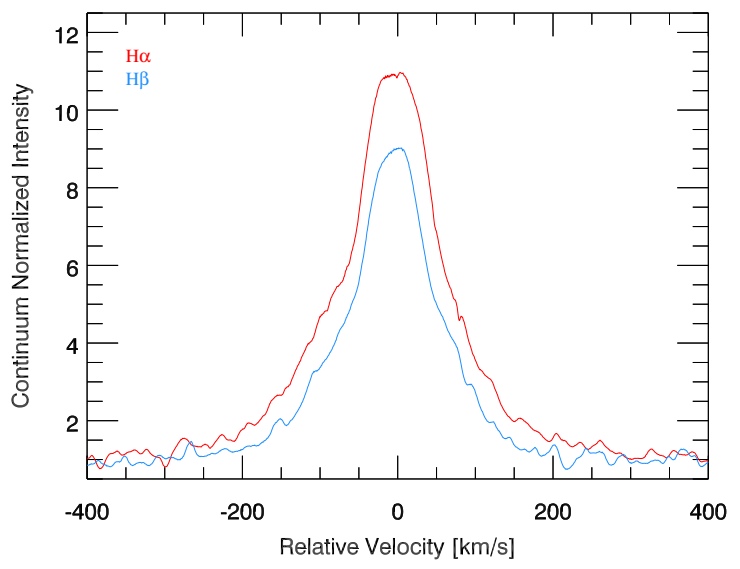

(c)

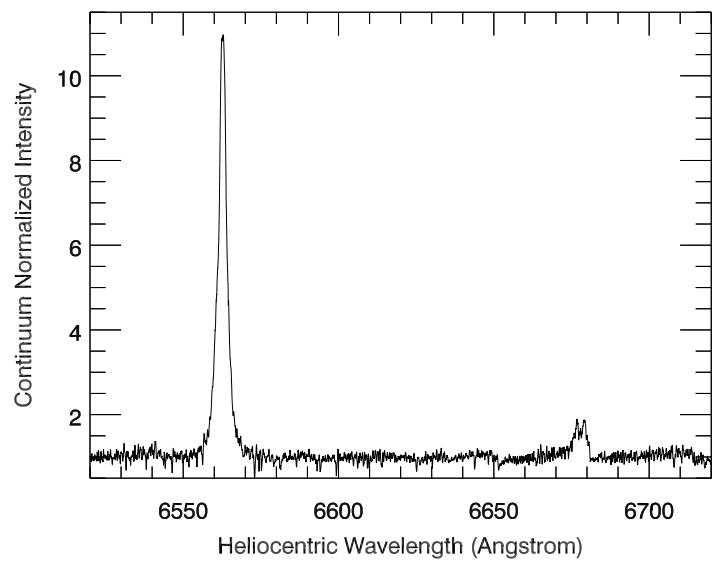

(b)

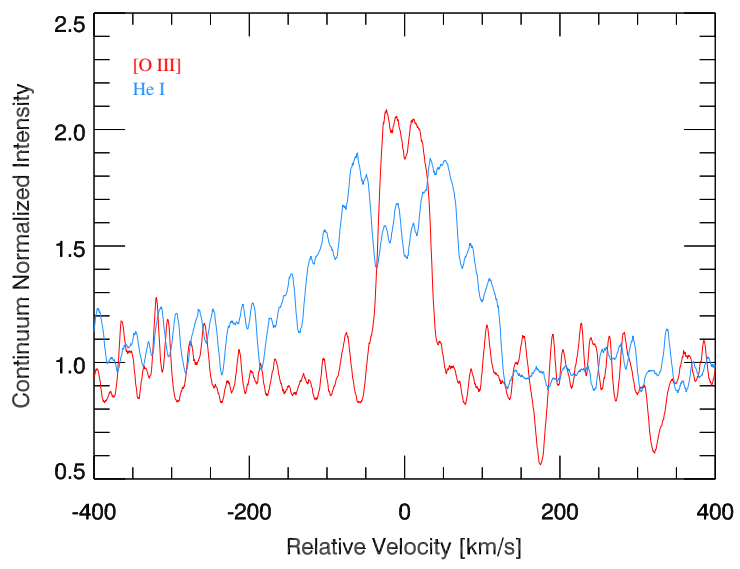

(d)

Figure 6. PEPSI continuum normalized spectra of T CrB. (a) A $200 \AA$ region near $\mathrm{H} \beta$, rest-wavelength in air of $4861.325 \AA$, that also shows the weaker [O III] line, rest wavelength in air of $5006.842 \AA$. The median continuum SNR is $\simeq 200$. (b) A $200 \AA$ region near $\mathrm{H} \alpha$, rest-wavelength in air of $6562.800 \AA$, that also shows emission from He I, $g_{k} * A_{k i}$ weighted average rest-wavelength in air $6678.152 \AA\left({ }^{1} \mathrm{P}_{o}-{ }^{1} \mathrm{D}\right)$ that clearly exhibits a double-horned structure. The median continuum SNR is $\simeq 500$. (c) The velocity structure of the hydrogen emission lines relative to their rest wavelengths. (d) The velocity structure of the [O III] and He I lines relative to their rest wavelengths. Note the change in relative intensity scale between the two figures. 